\title{
PCOS and uveitis: a case report
}

\begin{abstract}
Polycystic ovary syndrome (PCOS), a common endocrinopathy in reproductive-aged women, is a disease often accompanied by significant inflammation throughout the body. For a condition which is so prevalent throughout the world, there is a great deal of information about it that still needs to be discovered. No current research has been performed to determine if PCOS and its inflammatory pathophysiology affects the eye, specifically the uvea. This case report presents an otherwise healthy 30 -year-old female with diagnosis of PCOS and 3 recurrent uveitis episodes within one year.
\end{abstract}

Volume 8 Issue 3 - 2018

\author{
Ryan Capouch, Caryn LaBuda \\ Midwestern University-Illinois, USA
}

Correspondence: Caryn LaBuda, Midwestern UniversityIllinois, 3450 Lacey Road, USA, Tel +6307434800 ,

Email clabud@midwestern.edu

Received: May 16, 2018| Published: June 13, 2018

\section{Introduction}

Uveitis is a condition of inflammation of the uveal tract of the eye. This inflammation can be located in the iris, ciliary body, or the choroid or any combination of the three. This inflammation can be considered idiopathic in approximately $50 \%$ of individuals if the uveitis is only affecting the iris or the ciliary body. (Kanski). Typically in a patient who has an anterior uveitis episode, the initial episode is considered idiopathic until proven otherwise. However if the patient gets another bout of anterior uveitis then the provider begins to question whether there is some type of underlying immunological component. (Kanski) Polycystic ovary syndrome (PCOS) is a disease characterized by hyperandrogenism, absent or sparse ovulation, and multiple cysts on or around the ovaries. ${ }^{1}$ It affects $4-10 \%$ of reproductive-aged women, making it one of the most common female endocrinopathies. ${ }^{1}$ Up to $70 \%$ of women diagnosed with PCOS also experience insulin resistance, which is argued to be either a contributing cause to or a resultant effect of the disease. ${ }^{1}$ Many in the scientific community have contested that PCOS is a disease of inflammation, characterized by increases in pro-inflammatory markers. ${ }^{1-5}$

Uveitis is characterized by the inflammation of the uvea. ${ }^{6}$ The patient is generally symptomatic and complains of pain, photophobia, and redness. ${ }^{6}$ Uveitis is a sight-threatening condition due to the threat of iris attachment to the posterior cornea or anterior lens. ${ }^{6}$ The disease can affect anterior chamber, posterior chamber, or both. ${ }^{6}$ It is usually treated with topical steroid and topical mydriatic to reduce inflammation and prevent damage to the uvea. ${ }^{7}$ The topical steroid is used to alleviate uveitis by reinforcing the blood-aqueous barrier and reducing inflammation. The mydriatic is used to "sling" the iris and prevent contraction of the iris sphincter and dilator muscles, which reduces pain. Uveitis is considered a pro-inflammatory condition and can be caused by a great deal of systemic conditions, including several autoimmune conditions such as ankylosing spondylitis or Crohn's disease. Autoimmune diseases can progress and relapse throughout an individual's lifetime and this includes their potential bouts of uveitis. In the case of autoimmune conditions, we know that due to the lack of recognition of self vs. non-self the body is in a high state of inflammation. Uveitis is caused by inflammation in the eye, and can be caused by an overall inflammatory response in the body.

Steroids are considered an immunosuppressant and a general antiinflammatory medication. Why does this happen? Perhaps the answer can be found by going back to the pharmacology of the steroid. Steroids inhibit the arachadonic acid pathway, which is the pro-inflammatory pathway. The steroids block the formation of prostaglandins and cyclooxygenases, allowing an overall anti-inflammatory effect throughout the body. In the specific case of steroids and their effects on the eyes, while suppressing the arachadonic acid pathway, they also help support the blood aqueous barrier in terms of not allowing the inflammation to transfer from the blood vessels into the aqueous chamber. After an extensive literature review, no study was found to contain research postulating a link between PCOS and uveitis. One study, performed in 2012, suggested chronic uveitis may in the future be treated with metformin due to its anti-inflammatory mechanisms of action. ${ }^{2}$ The case report below presents a patient in otherwise good health with diagnosis of PCOS and three episodes of uveitis in one year. We aim to examine potential links between the inflammation process in PCOS and the inflammation process in uveitis. Based on knowledge of the pharmacokinetics of metformin, ${ }^{8}$ it is also possible that there may be a reduction of symptoms of uveitis in patients taking metformin.

\section{Case report}

A 30 year old Caucasian female professor presented to the clinic with a chief complaint of eye pain. This eye pain is qualified as a sharp, stabbing eye pain that is unrelenting. This eye pain is not alleviated by artificial tears or by Tylenol. The pain is rated as a $9 / 10$ on the pain scale. This pain is localized to the right eye only. She reports that this pain is impairing her ability to perform her job, such as grading papers. The nature of onset was sudden and has progressed over the last 6 hours. Light seems to exacerbate the symptoms for this patient. She reports that this has happened before, at least two times in the last year. Each one of these times, the patient was treated with Pred-Forte and the issue resolved with no sequelae. The Pred-Forte was prescribed for the patient in a traditional tapering format, where the patient was on Pred-Forte qid $\mathrm{x} 1$ day, tid $\mathrm{x} 1$ day, bid $\mathrm{x} 1$ day, and qd $x 2$ days.

Medical history for this patient is significant for polycystic ovarian syndrome (PCOS), but is not significant for any other findings. She is currently taking Metformin $850 \mathrm{mg}$ qd po, Metoprolol $50 \mathrm{mg}$ bid po, Vitamin D $2000 \mathrm{IU}$ qd po, Spironolactone $50 \mathrm{mg}$ bid po, and Mononessa qd po. This patient is allergic to sulfa drugs. Family medical history is significant for hypertension in both her mother and father, but no other significant history exists. Best corrected visual acuity at distance was 20/30 OD and 20/20 OS. Her best corrected visual acuity at near was 20/20 OD, OS. Her pupils were equal, round, reactive to light, with no signs of an afferent pupillary defect.

Her extraocular muscles and confrontation fields were within normal limits. Slit lamp examination revealed $1+$ cells and flare OD 
in the anterior chamber and a deep and quiet anterior chamber OS. There were no other significant findings on slit lamp examination. Intraocular pressure for this patient was $12 \mathrm{mmHg}$ OD and $14 \mathrm{mmHg}$ OS, measured by Goldman applanation tonometry. The patient was dilated and her dilated fungus examination was unremarkable bilaterally with C/D ratios of $0.30 / 0.30 \mathrm{OD}$, OS

Due to the fact that this is not the patient's first anterior uveitis, laboratory tests were ordered for this patient. Since the patient has a nonspecific endocrine disorder, a variety of laboratory tests were ordered in order to ensure an appropriate diagnosis and treatment for this patient long term. Please see the below table for the laboratory results and their reference ranges.

\begin{tabular}{|c|c|c|}
\hline Laboratory tests: & Results & Reference ranges \\
\hline Glucose & $80 \mathrm{mg} / \mathrm{dL}$ & 65-II5 (fasting) \\
\hline Urea nitrogen & II mg/dL & $5-24$ \\
\hline Creatinine & $0.8 \mathrm{mg} / \mathrm{dL}$ & $0.7-1.4$ \\
\hline Sodium & $141 \mathrm{meq} / \mathrm{L}$ & $|36-| 46$ \\
\hline Potassium & $4.0 \mathrm{meq} / \mathrm{L}$ & $3.7-5.3$ \\
\hline Chloride & $98 \mathrm{meq} / \mathrm{L}$ & $101-111$ \\
\hline $\mathrm{CO} 2$ & $18 \mathrm{meq} / \mathrm{L}$ & $2|-3|$ \\
\hline Calcium & $10 \mathrm{mg} / \mathrm{dL}$ & $8.5-10.2$ \\
\hline HgbAlc & $5.00 \%$ & $4.0-6.0$ \\
\hline RBC & $4.78 \mathrm{M} / \mathrm{mL}$ & $4.2-5.4$ \\
\hline $\mathrm{Hgb}$ & $13.9 \mathrm{~g} / \mathrm{dL}$ & $12.0-15.5$ \\
\hline Hct & $41.70 \%$ & $36-48$ \\
\hline MCV & $87 \mathrm{fL}$ & $80-103$ \\
\hline $\mathrm{MCH}$ & $29.1 \mathrm{pg}$ & $26-34$ \\
\hline $\mathrm{MCHC}$ & $33.3 \mathrm{~g} / \mathrm{dL}$ & $30-37$ \\
\hline RDW-CV & $13.00 \%$ & II.5-14.5 \\
\hline WBC & $12.9 \mathrm{~K} / \mathrm{mL}$ & $3.9-11.0$ \\
\hline Neutrophil \% & $73 \%$ & $49-78$ \\
\hline Lymphocyte \% & $22 \%$ & $20-45$ \\
\hline Monocyte \% & $5 \%$ & $3.0-9.5$ \\
\hline Eosinophil \% & $0 \%$ & $0.0-4.0$ \\
\hline Basophil \% & $0 \%$ & $0.0-3.0$ \\
\hline Platelet & $443 \mathrm{~K} / \mathrm{mL}$ & $130-400$ \\
\hline DHEA-S: & 773.4 & \\
\hline Cortisol PM: & 13 & $2.3-11.9$ \\
\hline Cortisol AM: & 34.2 & $6.2-19.4$ \\
\hline LAP score: & $|5|$ & $25-130$ \\
\hline HLA-B27 & Negative & \\
\hline TB by PPD & Negative & \\
\hline TB by QuantiFERON Gold & Negative & \\
\hline ANA & Negative & \\
\hline Rheumatoid Factor & Negative & \\
\hline ESR & 36 & $0-29$ \\
\hline High sensitivity CRP & 12.0 mg/L & $<3.0$ \\
\hline
\end{tabular}

\section{Discussion}

This patient based on her case history clearly has polycystic ovarian syndrome. However, what is important to note about this particular patient is that her inflammatory markers are exceedingly high even compared to additional PCOS patients. What does this mean for the patient? Clearly, there is a great deal of inflammation in the body. In this patient's case there is a logical reason for her inflammation: she has an underlying autoimmune type reaction throughout the body. Based on her blood work the patient has elevated cortisol, erythrocytes sedimentation rate, and C-reactive protein. This indicates that the patient has inflammation throughout the body, which could easily be transferable to the vessels of the anterior segment of the eye. Her medications are reasonable with the treatment of PCOS. However, all of these medications are off-label use, which means that they are not recognized by the FDA for actually treating this condition. The birth control is treating the androgenic effects of the PCOS; the metformin is used to treat insulin resistance, but this patient does not have any signs of insulin resistance; the metformin in this case is being used as anti-inflammatory medication to suppress the overall pro-inflammatory conditions this patient has going on in the body. The vitamin D is not being used in the management of PCOS and has no effect on the disease progression. Spironolactone is used as an off label medication to treat the hirsutism of the patient. Spironolactone is traditionally used as a mild anti-hypertensive agent, but the side effect of this particular medication is the diminished amount of hair growth across the body. The patient is on metoprolol for hypertensive treatment, which is often a common co-morbidity to PCOS. Metoprolol is in the B-blocker class of medications and is not being used to treat anything specifically related to the PCOS.

Realistically, the treatments that this patient is on are not treating the underlying cause, but rather are just masking the symptoms for this patient so that she can carry on with her activities of daily living. This particular condition is an unfortunate one because one of the main suggestions for this condition is weight loss in the patient, but the more the patient tries to lose weight the more symptomatic the patient typically becomes because of the overall increased inflammation in the body. While this blood panel may seem comprehensive or even over-kill in some regards, this patient with the three recurrent bouts of uveitis in such a small period of time needed to be evaluated for any other underlying conditions that she might have so that they could be systemically treated and managed. We chose to order an inflammatory panel as well because of the known inflammation in the body typically caused by PCOS. Pain with uveitis is difficult to assess because depending how much of the iris and ciliary body are involved and depending on how much the pupil needs to dilate or constrict depends on the amount of pain that a patient will feel. In the case of chronic anterior uveitis similar to other chronic conditions while the patient will feel pain, they will feel less pain than someone with an acute onset of anterior uveitis, which is what this patient had. The patient is a reliable patient so it is doubtful that this patient is lying about the status of the pain. The patient did not even want to take pain killers for the pain so there was no question of this patient fabricating pain in a drug seeking fashion.

Typically in uveitis the patient experiences difficulty with vision at near because the pupil is trying to constrict. This patient was experiencing pain at distance vision, which at first glance doesn't seem like a typical symptom. However, when further evaluating this patient's activities that she had engaged in most of the day she was grading and working on her computer the majority of the day, which 
means that her pupils were likely constricted the majority of the day, and then when she tried to do distance tasks and her pupil was trying to dilate, she experienced pain. This case presents several confounding factors, which lead to the plausibility of PCOS playing a contributing role in the patient's uveitis episodes. At first glance, it may seem her uveitis could be written off to an idiopathic cause. However, the patient's otherwise unremarkable medical history, combined with extensive blood work containing several pro-inflammatory results, suggests that PCOS may be a significant factor in determining the etiology of her condition.

The patient had been seen by several gynaecologists, endocrinologists, and women's health specialists. She was prescribed several medications to control her symptoms for polycystic ovary syndrome. Considering research on metformin's anti-inflammatory properties, it may be possible that her use of metformin has lessened the degree of chronic uveitis and the frequency of episodes. Future studies are certainly indicated to determine how PCOS affects the uvea. Studies may also be beneficial in determining whether metformin could be used as a long-term treatment to reduce frequency of episodes and severity of uveitis. Determining how the inflammation of PCOS affects the uvea could change how practitioners treat and manage uveitis in reproductive-aged women. Management of this particular uveitis case may prove more difficult, as several inflammatory factors will increase risk of rebound inflammation. However, the general course of treatment for uveitis, paired with continued dosage of metformin, will significantly help the patient with her symptoms and reduce the risk of sight-threatening complications.

\section{Conflict of interest}

The author declares that there is no conflict of interest.

\section{References}

1. González F, Rote NS, Minium J, et al. Evidence of proatherogenic inflammation in polycystic ovary syndrome. Metabolism. 2009;58(7):954 962.

2. Duleba AJ, Dokras A. Is PCOS an inflammatory process? Fertil Steril. 2012;97(1):7-12.

3. Escobar-Morreale HF, Luque-Ramírez M, González F. Circulating inflammatory markers in polycystic ovary syndrome: A systematic review and metaanalysis. Fertil Steril. 2011;95(3):1048-1058.e2.

4. González F. Inflammation in polycystic ovary syndrome: Underpinning of insulin resistance and ovarian dysfunction. Steroids. 2012;77(4):300305 .

5. Pan J, Kapur M, McCallum R. Noninfectious immune-mediated uveitis and ocular inflammation. Curr Allergy Asthma Rep. 2014;14(1):1-8.

6. DickA.Pathophysiologyofuveitis.ActaOphthalmologica.2016;94(S256).

7. Sugita S, Shimizu J, Makabe K, et al. Inhibition of T cell-mediated inflammation in uveitis by a novel anti-CD3 antibody. Arthritis Res Ther. 2017;19(1):176.

8. Kalariya NM, Shoeb M, Ansari NH, et al. Antidiabetic drug metformin suppresses endotoxin-induced uveitis in rats. Invest Ophthalmol Vis Sci. 2012;53(7):3431-3440.

\section{Acknowledgements}

None. 